\title{
Tuned Mass Dampers for the Mitigation of Impulsive Ground Motions
}

\author{
E. Matta, R. Ceravolo, A. De Stefano \\ A. Quattrone and L. Zanotti Fragonara \\ Department of Structural, Geotechnical and Building Engineering \\ Politecnico di Torino, Turin, Italy
}

\begin{abstract}
The performance of tuned mass dampers (TMDs) diminishes as the input duration shortens. As a result, they are not recommended for use against short-duration, pulse-like ground motions, such as those occurring in near-field (NF) zones in the presence of forward-directivity or fling-step effects. Yet a systematic assessment of this control loss is still missing. In this paper, a recent analytical model of ground motion pulses is applied to the design and evaluation of TMDs against impulsive earthquakes. Based on this model, first a new optimization method is introduced as an alternative to the classical $\mathrm{H}_{\infty}$ approach. Then the two strategies are tested on single- and multi- degrees-of-freedom linear structures subject both to analytical pulses and to a large set of NF records possessing pulse-like features. The resulting statistical evaluation, expressed by percentile response spectra, shows the advantages and disadvantages of a pulse-oriented TMD design, and improves the general understanding of TMDs effectiveness under impulsive ground motions.
\end{abstract}

Keywords: earthquake engineering, structural control, seismic design, damping, earthquake resistant structures, ground motion, impulsive loads, response spectra.

\section{Introduction}

Passive tuned mass dampers (TMDs) are widely used in Civil Engineering to mitigate vibrations induced by quasi-stationary dynamic loads (winds, sea waves, pedestrians), but their seismic performance is known to depend on ground shaking properties. Requiring the motion of the primary structure to react with, TMDs prove effective against long-duration, narrow-band ground motions, but may fail in reducing the peak response to pulse-like earthquakes [1].

One typical example of pulse-like earthquakes is given by near-field (NF) ground motions. Ground shaking near a fault rupture may be characterized by a shortduration impulsive motion that exposes structures to high input energy at the 
beginning of the record. This pulse-type motion can be explained through the concepts of "forward-directivity" and "fling-step" effects [2]. The former is due to the fault rupture propagating towards the site at a velocity close to the shear wave velocity, causing most of the seismic energy to reach the site within a short time, in the form of a large energy pulse at the beginning of the record, mostly oriented in the fault-normal direction. The latter is due to permanent ground displacements accumulating at the site as a result of tectonic movements. Both effects may result in large-amplitude, long-period pulses in the velocity and displacement time histories, particularly challenging for the structural safety of long-period structures.

Many studies have been devoted to improving the performance of structures exposed to NF ground motion. The use of simplified analytical models of ground motion pulses may prove a valid tool for the systematic design and assessment of seismo-protective systems. Several models are available for this purpose [3,4]. Most of these models, based on discontinuous functions, are not ideal for extensive parametric studies. Continuous models have been proposed recently which sound more promising for that purpose [5]. One of them is the velocity pulse model suggested by $\mathrm{He}$ and Agrawal (2008) to study the performance of supplemental viscous dampers on a base-isolated building [6]. Based on Belarge model and essentially consisting of an amplitude modulated sinusoid, this model can successfully depict both buildup and decaying phases of recorded ground motions, beyond possessing two valuable properties: (a) existence of a closed-form solution for a single-degree-of-freedom (SDOF) structure subject to the ground model; (b) existence of a counterpart frequency domain and state-space domain expression that can be used in the design of seismic protective systems.

In this paper, in order to show the possible advantages of a pulse-oriented TMD design, this model is applied to the optimization and evaluation of TMDs against impulsive earthquakes. A new "pulse design" is introduced as an alternative to the classical $\mathrm{H}_{\infty}$ approach, and the two strategies are tested on SDOF and multi-degreesof-freedom (MDOF) linear structures subject to both analytical pulses and NF real records. The resulting statistical assessment, expressed by percentile response spectra, shows the pros and cons of a pulse-oriented TMD design and improves the general understanding of TMD performance under impulsive ground motions.

\section{Design of TMDs under pulse-like ground motions}

\subsection{The analytical pulse model}

The ground velocity analytical model proposed in [6] is defined as

$$
\dot{u}_{p}(t)=C t^{n} e^{-a t} \sin \left(\omega_{p} t+v\right), \quad t \geq t_{0}
$$

where $\omega_{p}=2 \pi / T_{p}=$ pulse circular frequency; $T_{p}=$ pulse period; $C=$ amplitude scaling factor; $v=$ phase angle of the sinusoidal component; $a=$ decay factor; $n=$ skewness parameter; and $t_{0}=$ beginning time of the pulse. 
This model is essentially an amplitude modulated sinusoid and can simulate recorded NF ground motions by selecting appropriate pulse parameters. The parameters $n$ and $a$, controlling the speed of, respectively, buildup and decay of the velocity pulse, can be alternatively expressed in terms of the number of cycles in the buildup phase, $N$, and the pulse decay ratio, $\zeta_{p}$ (which has the same physical meaning as the parameter $\zeta_{g}$ in the Kanai-Tajimi model), according to

$$
N=n /\left(a T_{p}\right) \text { and } \zeta_{p}=a / \sqrt{a^{2}+\omega_{p}^{2}}
$$

The model degenerates into a sinusoid as $N$ goes to infinity and/or $\zeta_{p}$ goes to zero.

Pulse parameters have been identified in [6] for 36 recorded NF ground motions through nonlinear least square regression. The same set of records, herein termed Set-36 for brevity, and their analytical approximations will be used herein to assess TMDs performance.

\subsection{TMD design}

Several methods exist to design a TMD on an SDOF structure of period $T$ and damping ratio $\zeta_{s}$ under ground motion. Denoting as mass ratio, $\mu$, the ratio of the TMD mass to the structural mass, and as frequency ratio, $r$, the ratio of the TMD natural frequency to the structural natural frequency, any design method will consist in: (a) arbitrarily fixing $\mu$ (based on costs/benefits considerations); and (b) accordingly selecting $r$ and the TMD damping ratio, $\zeta_{t}$, so that the 2-DOF combined system, obtained mounting the TMD on the SDOF structure, shall be optimum in terms of some predefined objective. Depending on the chosen objective, various optimization techniques are available. The most widespread techniques minimize some norm of a given input-output steady-state transfer function of the combined system, typically chosen as the one from the ground acceleration to the structural displacement relative to the ground, here denoted as $T F$. Depending on the chosen norm, these techniques can be mainly distinguished into the $\mathrm{H}_{2}$ (norm) design [7] and the $\mathrm{H}_{\infty}$ (norm) design [8]. Of course, these criteria assume the ground motion as a stationary excitation, irrespectively of any short-duration feature.

In this study, two methods are compared. The first one is the classical $\mathrm{H}_{\infty}$ design which, for the given $\zeta_{s}$ and $\mu$, selects $r$ and $\zeta_{t}$ so as to minimize the $\mathrm{H}_{\infty}$ norm of the acceleration-to-displacement transfer function $T F$ [10]. This equals to minimizing the maximum steady-state structural displacement amplitude under a sinusoidal acceleration time-history having constant amplitude and any possible frequency.

The second one is a new pulse optimization, designated as $\mathrm{H}_{p}$ design, which degenerates into the $\mathrm{H}_{\infty}$ design if the ground motion degenerates into a sinusoid, but differs from the $\mathrm{H}_{\infty}$ design otherwise. In the $\mathrm{H}_{p}$ design, the ground acceleration is defined by Eqs. (2) and (3), posing $v=0$ and $t_{0}=0 \mathrm{~s}$, and scaled to have a unitary maximum value; so the input signal depends exclusively on the pulse frequency, $\omega_{p}$, and on the pair of buildup and decay parameters, $N$ and $\zeta_{p}$. Then, for any assigned 
pair $N$ and $\zeta_{p}$, the "pulse transfer function", $T F_{p}$, is evaluated for the combined system, reporting the pulse frequency $\omega_{p}$ in the abscissas and the corresponding peak structural displacement in the ordinates. The new $\mathrm{H}_{p}$ design consists in numerically finding the optimal TMD parameters, $r$ and $\zeta_{t}$, which minimize the maximum of $T F_{p}$, for the assigned values of $\zeta_{s}, \mu, N$ and $\zeta_{p}$.

In Figure 1, assuming $\zeta_{s}=2 \%, \mu=10 \%, N=1.0$, and $\zeta_{p}=0.4$, the two design methods are compared on a SDOF structure of period $T=1 \mathrm{~s}$. The numerical optimization provides the following TMD parameters: $r=0.873$ and $\zeta_{t}=0.194$ for the $\mathrm{H}_{\infty}$ design, $r=0.859$ and $\zeta_{t}=0.026$ for the $\mathrm{H}_{p}$ design. On the left, the steadystate transfer functions $T F$ are plotted for, respectively, the uncontrolled structure, the $\mathrm{H}_{\infty}$-controlled structure, and the $\mathrm{H}_{p}$-controlled structure. On the right, the corresponding pulse transfer functions $T F_{p}$ are reported. A trade-off clearly emerges between the two design methods: the $\mathrm{H}_{\infty}$ design, optimal against sine inputs, loses effectiveness under pulse-like inputs; the $\mathrm{H}_{p}$, optimal for pulse mitigation, is severely impaired in steady-state terms. The reason is that, in order to minimize the peak response to the pulse load, the $\mathrm{H}_{p}$ design decreases TMD damping ratio with respect to the $\mathrm{H}_{\infty}$ design, at such an extent that the dotted curve on the left looks more like Frahm's undamped vibration absorber than like Den Hartog's tuned mass damper. The reduced damping allows the TMD to more rapidly respond to the impulsive load, but hinders it in reducing the two peaks of the steady-state transfer function. Also, the reduced damping has a second inconvenient (not apparent in Figure 1), of diminishing TMD capability to control the post-peak, free-decay response of the structure.

The two mentioned drawbacks, together with the need to rely on predictions of pulses features, pose the question of the true advantage of a pulse-oriented optimization, whose merits over a classical $\mathrm{H}_{\infty}$ design seem substantially circumscribed. An answer will be attempted in the next section.
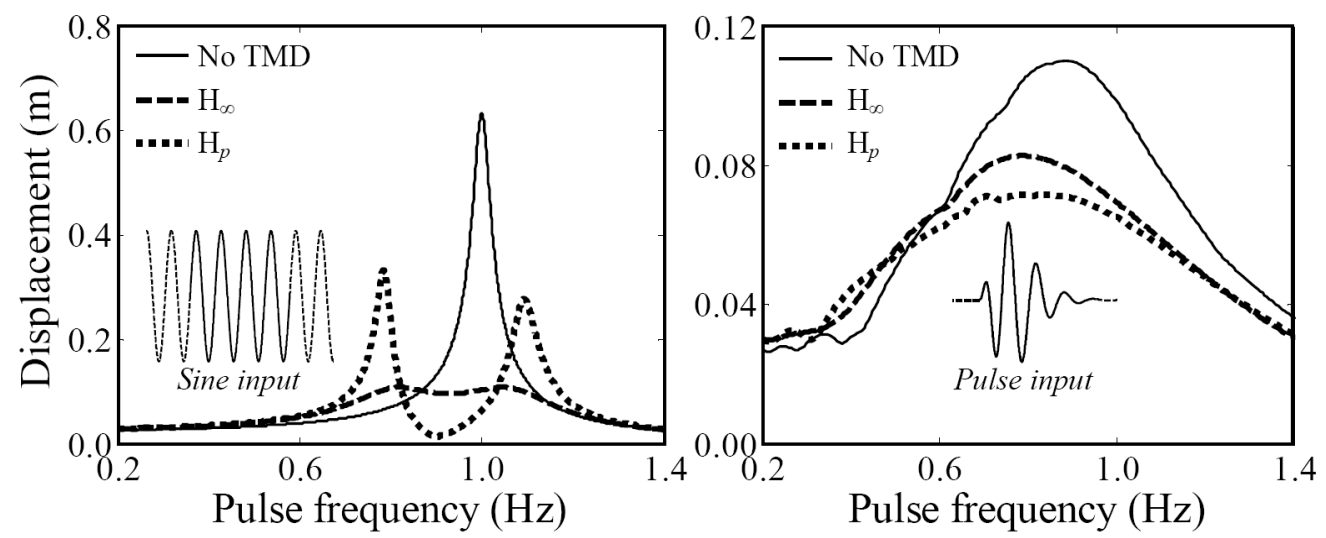

Figure 1: $\mathrm{H}_{\infty}$-and $\mathrm{H}_{p}$-designed TMD $(\mu=10 \%)$ on an SDOF structure: (left) TF (sine input); (right) $T F_{p}$ (pulse input) 


\section{Performance of TMDs on SDOF structures under pulse-like ground motions}

In this section, the performance of $\mathrm{H}_{\infty}$-designed and $\mathrm{H}_{p}$-designed TMDs on SDOF structures is evaluated under, respectively, analytical pulses and real records.

\subsection{Analytical pulses}

Two performance measures are used herein to evaluate TMDs against the analytical pulses in Eq. (1): the peak displacement ratio, $R_{d}$, obtained dividing the maximum of the controlled $T F_{p}$ by the maximum of the uncontrolled $T F_{p}$; and the root-meansquare (RMS) velocity ratio, $R_{v}$, obtained in the same way but replacing the peak of the displacement with the RMS of the velocity.

Two mass ratios are considered, namely $\mu=10 \%$ and $\mu=50 \%$. These percentages, although sensibly larger than the few percents commonly employed in wind or traffic mitigation, are however not at all unrealistic, as it will be shown in the next sections.

For each $\mu$, assuming $\zeta_{s}=2 \%$, the $\mathrm{H}_{p}$ optimization is repeated for a number of $N$ $\zeta_{p}$ pairs in the range $[0.2-5.0,0.0-0.8]$. Each $N-\zeta_{p}$ pair (univocally defining a ground motion) produces a different couple of TMD optimal parameters, $r$ and $\zeta_{t}$. The resulting "maps" are reported in Figure 2 for $\mu=50 \%$. The $\mathrm{H}_{\infty}$ counterpart of these maps is obviously a single couple of optimal parameters, which for $\mu=50 \%$ happens to be $r=0.556$ and $\zeta_{t}=0.408$.
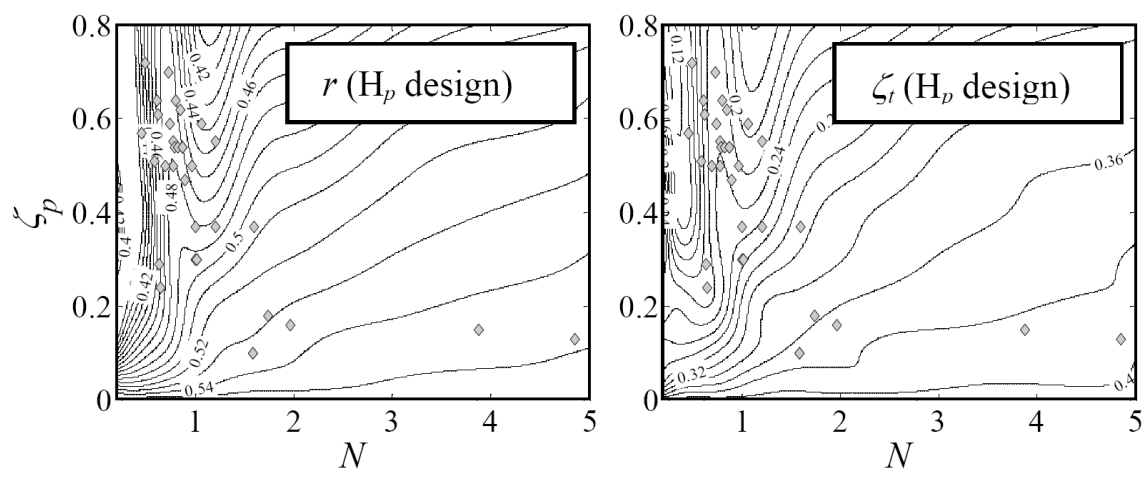

Figure 2: Dependence of TMD optimal parameters $r$ and $\zeta_{t}$ on pulse parameters $N$ and $\zeta_{p}$ according to the $\mathrm{H}_{p}$ design $(\mu=50 \%)$

Then, for each $N-\zeta_{p}$ pair, the response ratios $R_{d}$ and $R_{v}$ are evaluated, alternatively adopting $\mathrm{H}_{\infty}$ or $\mathrm{H}_{p}$ as the design criterion. This results in four further maps for each $\mu$. It is worth noticing that, according to such procedure, the $\mathrm{H}_{p}$-designed TMD is tested under exactly the same motion for which it has been optimized. Results are reported in Figure 3 for $\mu=50 \%$. 

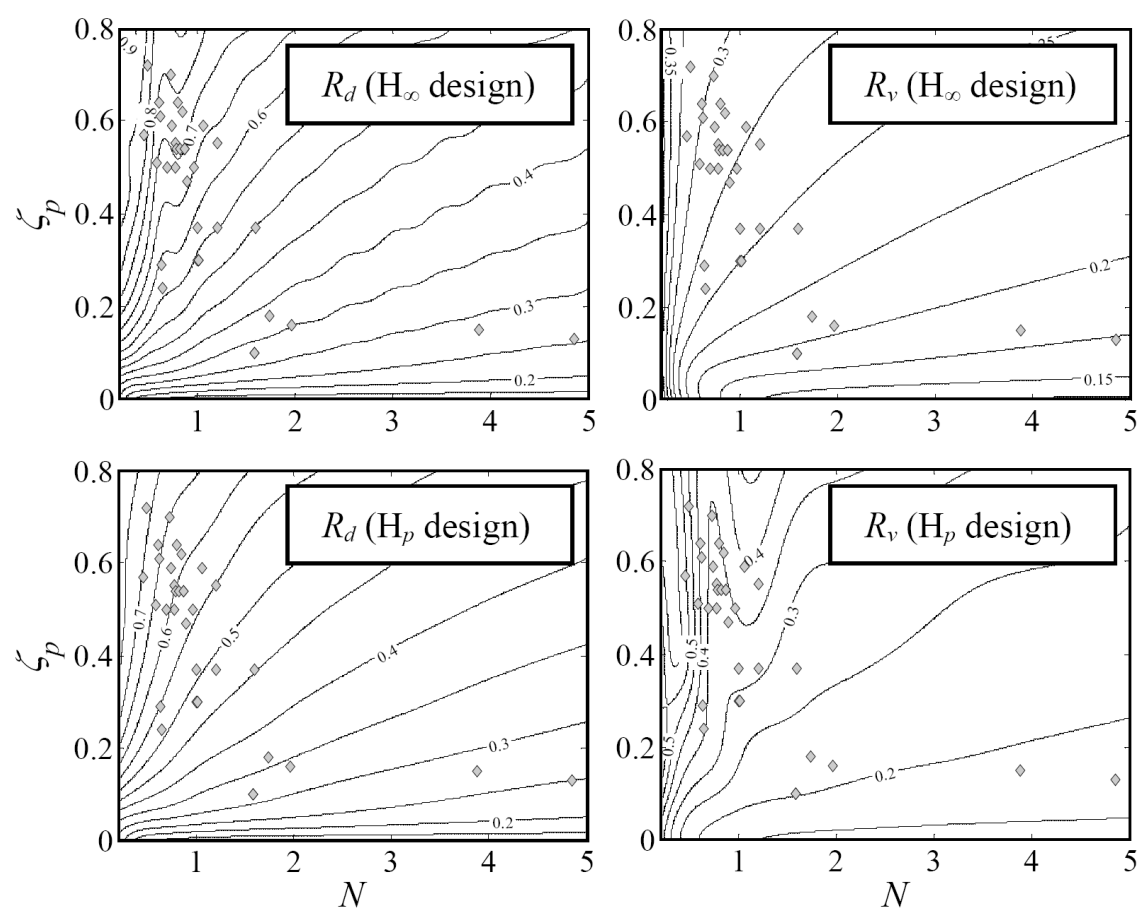

Figure 3: Dependence of $R_{d}$ and $R_{v}$ on pulse parameters $N$ and $\zeta_{p}$ according to, alternatively, the $\mathrm{H}_{\infty}$ and the $\mathrm{H}_{p}$ design $(\mu=50 \%)$

In each of the six said maps, 36 markers identify the $N-\zeta_{p}$ pairs which best fit, according to [6], the records in Set-36. The markers are mostly clustered in the range $[0.6-1.2,0.2-0.7]$, their mean value being at $N=1.134$ and $\zeta_{p}=0.432$.

In the said maps, the upper left corner $\left(N=0.2, \zeta_{p}=0.8\right)$ and the lower right corner $\left(N=5, \zeta_{p}=0\right)$ represent, respectively, the most impulsive input and a perfect sinusoid. Actually, the entire lower side of the map $\left(\zeta_{p}=0\right)$ represents a perfect sine. This explains why, moving rightwards and/or downwards along the maps, the $\mathrm{H}_{p}$ design tends to the $\mathrm{H}_{\infty}$ design and the response ratios $R_{d}$ and $R_{v}$ decrease.

Around the central cluster of markers, the $\mathrm{H}_{p}$ design enforces a reduction of $r$ and, especially, of $\zeta_{t}$, with respect to the $\mathrm{H}_{\infty}$ design. These reductions improve peak control but significantly worsen RMS control. In any case, no matter what the design method, TMD performance around that cluster is severely diminished with respect to steady-state performance. Since the $\mathrm{H}_{p}$ design is by definition optimal in peak terms, large mass ratios appear necessary to satisfactorily control pulse-like excitations.

Far from that cluster, other markers are located in a more favorable position, where, thanks to larger values of $N$ and/or lower values of $\zeta_{p}$, TMD performance improves and differences between the two methods are smaller.

The large dispersion of the markers within the maps makes the $\mathrm{H}_{p}$ design dependent on the ability to predict the main pulse characters of future events. Since relying on site-specific accurate predictions is unrealistic, the maps should be interpreted, rather than as an operative design tool, as: (a) the representation of the 
trade-off between $\mathrm{H}_{p}$ and $\mathrm{H}_{\infty}$ design as well as between peak and RMS performance; (b) the proof that reducing $r$ and $\zeta_{t}$ improves peak response mitigation under impulsive quakes; (c) the measure of the largest possible peak reduction achievable with a TMD.

Results are summarized, for both $\mu=10 \%$ and $\mu=50 \%$, in Table 1 , which reports the optimal TMD parameters and the consequent response ratios corresponding to the 36 markers, in mean and maximum terms. In addition to the $\mathrm{H}_{\infty}$ and the $\mathrm{H}_{p}$ methods a third method is introduced, named $\mathrm{H}_{p}{ }^{*}$, which is nothing but the $\mathrm{H}_{p}$ design applied admitting that no exact prediction is possible on the future event, and therefore assuming $N=1.0$ and $\zeta_{p}=0.4$ (roughly corresponding to a kind of average pulse) at the design stage. In other words, whilst $\mathrm{H}_{p}$ is an unrealistic solution, merely showing the theoretical upper bound of TMD pulse performance, $\mathrm{H}_{p}{ }^{*}$ is a viable design strategy, in which a reduction of $r$ and $\zeta_{t}$ can be enforced at the design stage as a means to improve peak performance, at the cost of diminishing RMS performance.

\begin{tabular}{|c|c|c|c|c|c|c|c|}
\hline \multirow{2}{*}{$\mu$} & Design & $r$ & $\zeta_{t}$ & \multicolumn{2}{|c|}{$R_{d}$} & \multicolumn{2}{|c|}{$R_{v}$} \\
\cline { 3 - 8 } & method & mean & mean & mean & $\max$ & mean & $\max$ \\
\hline \multirow{3}{*}{$10 \%$} & $\mathrm{H}_{\infty}$ & 0.873 & 0.194 & 0.732 & 0.878 & 0.384 & 0.443 \\
& $\mathrm{H}_{p}$ & 0.834 & 0.033 & 0.658 & 0.804 & 0.583 & 0.842 \\
& $\mathrm{H}_{p}{ }^{*}$ & 0.859 & 0.026 & 0.691 & 0.806 & 0.555 & 0.627 \\
\hline \multirow{3}{*}{$50 \%$} & $\mathrm{H}_{\infty}$ & 0.556 & 0.408 & 0.607 & 0.827 & 0.264 & 0.315 \\
& $\mathrm{H}_{p}$ & 0.480 & 0.252 & 0.541 & 0.709 & 0.326 & 0.502 \\
& $\mathrm{H}_{p}{ }^{*}$ & 0.476 & 0.243 & 0.553 & 0.721 & 0.324 & 0.357 \\
\hline
\end{tabular}

Table 1: Optimal TMD parameters and consequent response ratios under the analytical pulses in Set-36

Table 1 reveals that:

(a) The new $\mathrm{H}_{p}{ }^{*}$ design seems a promising alternative to the classical $\mathrm{H}_{\infty}$ design. Preference should be given to $\mathrm{H}_{p}{ }^{*}$ or to $\mathrm{H}_{\infty}$ according to how sensible the designer is to the two objectives of peak and RMS response reductions. As far as $R_{d}$ is concerned, $\mathrm{H}_{p}{ }^{*}$ surpasses $\mathrm{H}_{\infty}$, not only in mean terms but especially in worst-case terms, showing an enhanced robustness against severe pulses. In this regard, passing from 0.878 to 0.806 ( $\max R_{d}$ for $\mu=10 \%$ ) actually improves effectiveness (meant as the complement to unity of the response ratio) from $1-0.878=12.2 \%$ to $1-0.806=$ $19.4 \%$, i.e. 1.6 times. On the other hand, $\mathrm{H}_{p}{ }^{*}$ is only slightly worse than the best possible pulse design, $\mathrm{H}_{p}$, showing that, even with no exact prediction of the pulse features, a pulse optimization is reasonable. As far as $R_{v}$ is concerned, conversely, $\mathrm{H}_{p}{ }^{*}$ worsens $\mathrm{H}_{\infty}$ as a result of the lesser damping, though at an extent which may be acceptable for $\mu=10 \%$, and certainly is for $\mu=50 \%$;

(b) Focusing on the $\mathrm{H}_{p}{ }^{*}$ design, and assuming $\mu=10 \%$, the effectiveness in mean terms equals $30.9 \%$ and $44.5 \%$, respectively for the peak displacement and the RMS velocity; assuming $\mu=50 \%$ the corresponding values grow to, respectively, $44.7 \%$ 
and $67.6 \%$. Then, increasing the mass ratio from $10 \%$ to $50 \%$ improves $\mathrm{TMD}$ effectiveness 1.45 times in peak terms and 1.52 times in RMS terms.

(c) A properly designed TMD with enough mass ratio can acceptably mitigate the effects of ground motion pulses.

These conclusions hold as long as the pulse model in Eq. (1) is used and the performance is evaluated in worst-case terms based on input-output transfer functions. In the next subsection, their validity will be verified on real ground motion records.

\subsection{Real records}

Dealing with real records requires a little change of perspective as well as of analysis tools. Transfer functions, implying the structure being assigned and the ground motion frequency $\omega_{p}$ being varied, are now replaced by response spectra, implying the structural period $T$ being varied and the ground motion being assigned. For all the ground motions in Set-36, response spectra are computed under both the real record and its analytical approximation, for the sake of comparison. Spectra are evaluated, for the SDOF structure having $\zeta_{s}=2 \%$ and $T$ in the $0-10 \mathrm{~s}$ range, in terms of peak relative displacement $d$, RMS relative velocity $v$, and peak absolute acceleration $A$. Both $\mu=10 \%$ and $\mu=50 \%$ are considered, and the three methods $\mathrm{H}_{\infty}, \mathrm{H}_{p}$ and $\mathrm{H}_{p}{ }^{*}$ are compared.

In Figure 4, for example, displacement spectra are computed under the SCS052 component of Northridge Earthquake, adopting the $\mathrm{H}_{p}^{*}$ design. The peculiar property of TMDs is apparent, of being advantageous on structures whose period is "tuned" to the ground motion and nearly useless elsewhere, i.e. of being seismically effective only when strictly needed. This property, intrinsic expression of robustness against the severity of seismic effects, results in fact in a "spectral-peak-smoothing effect" of TMDs against earthquake loadings.
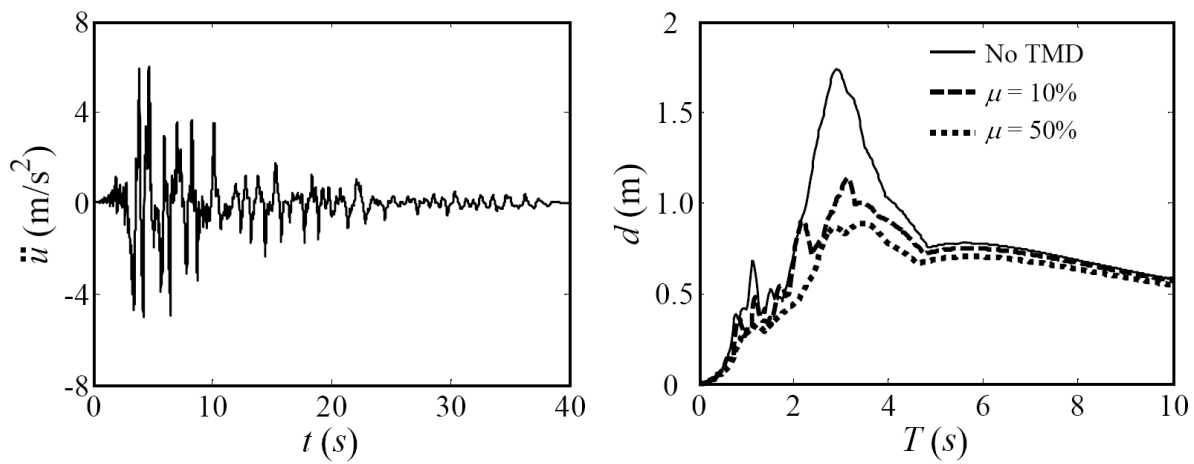

Figure 4: Northridge Earthq., Comp. SCS052: (left) record; (right) displacement spectra according to the $\mathrm{H}_{p}{ }^{*}$ design

To account for such effect, once the spectra are computed for the set of the 36 real records, for each structural period $T$ the 36 spectral ordinates are sorted in ascending order, then "percentile response spectra" are deduced connecting, period after 
period, the spectral ordinates of equal rank. Implicit in this statistical procedure is the assumption of equal-probability of the 36 ground motions. The procedure is then repeated for the set of the 36 analytical approximations. Results are exemplified for the 95\% percentile in Figure 5, which refers to an $\mathrm{H}_{p}{ }^{*}$-designed TMD having $\mu=$ $50 \%$. The corresponding "percentile response ratio spectra", obtained dividing the controlled by the uncontrolled percentile response spectra, are then reported (extended to $\mu=10 \%$ ) in Figure 6. Figure 6 also includes spectra corresponding to an MDOF structure, which will be explained in the next section.
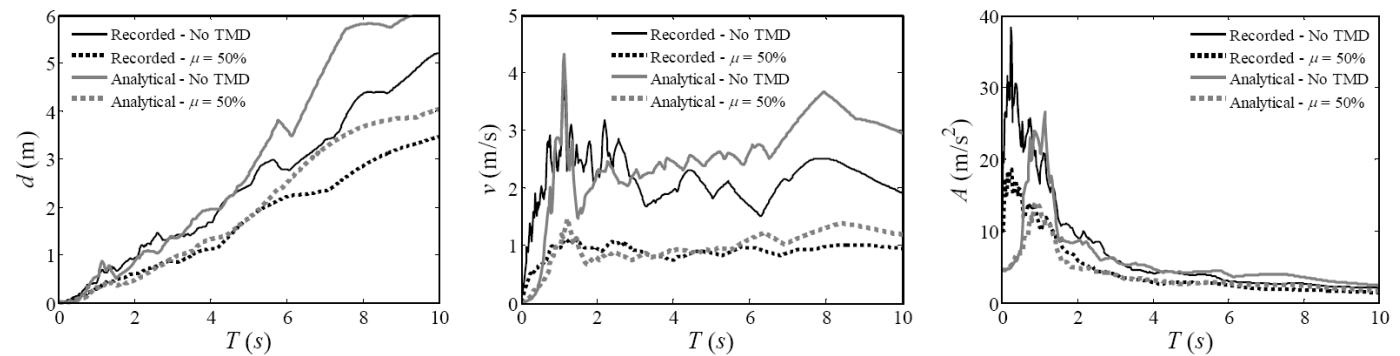

Figure 5: 95\% percentile response spectra under the real records in Set-36 and their analytical approximations, for a SDOF structure with or without an $\mathrm{H}_{p}{ }^{*}$-designed TMD $(\mu=50 \%)$
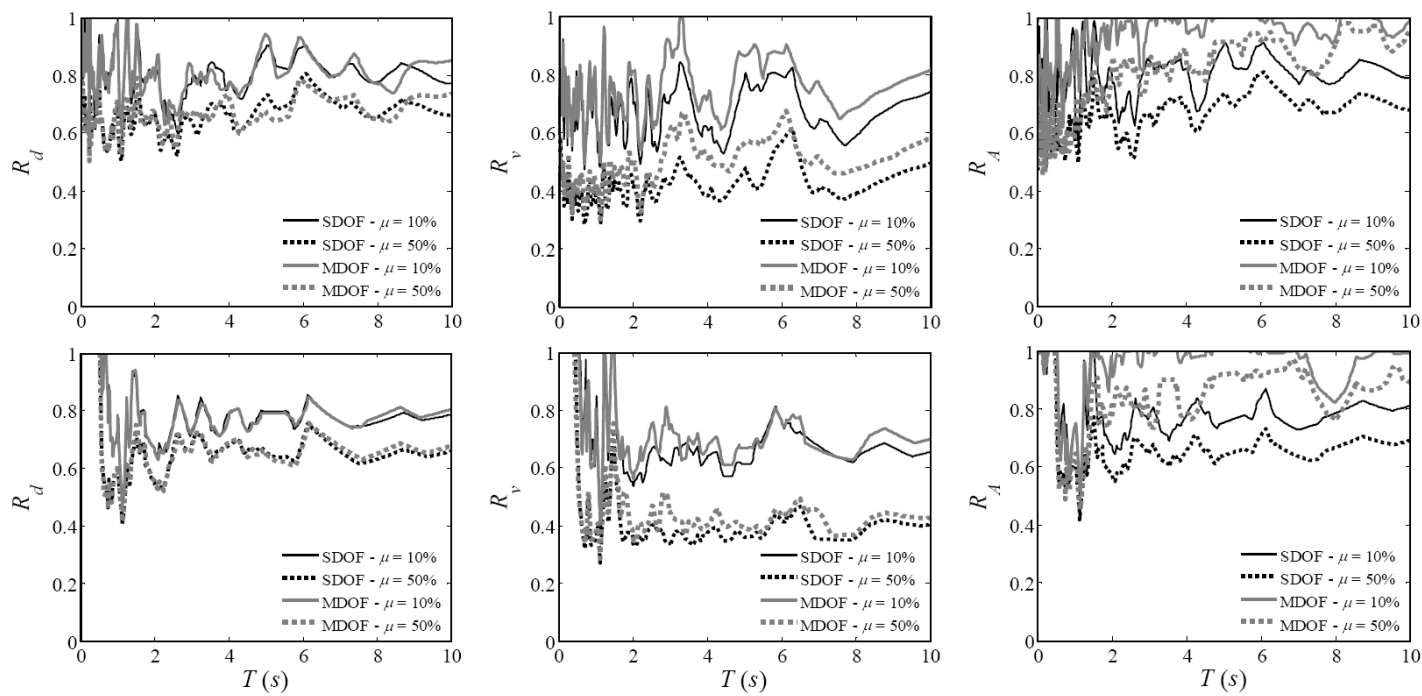

Figure 6: 95\% percentile response ratio spectra under the ground motions in Set-36, for SDOF and MDOF structures controlled through an $\mathrm{H}_{p}{ }^{*}$-designed TMD $(\mu=10 \%$ or $\mu=50 \%$ ): (up) real records; (down) analytical approximation

In Figure 5, coherently with the results obtained in [6] for an uncontrolled 5\%damped SDOF structure, the spectra from real records and those from analytical pulses appear to match well for approximately $T>1.5 \mathrm{~s}$, whereas for $T<1.5 \mathrm{~s}$ the 
fitted analytical model proves inadequate because of unmodeled high frequency components.

In Figure 6, as far as the SDOF structure is concerned (black curves), real and analytical spectra match on average quite well for $T>1.5 \mathrm{~s}$. An acceptable matching can be recognized, at some extent, for $T$ as low as about $0.6 \mathrm{~s}$. Below this threshold, instead, discrepancies become unacceptable, with the TMD still effective against real records (except below 0.1s, due to the well-known inadequacy of TMDs to seismically control "rigid" structures) while even detrimental against their analytical approximations (because of their lack of high-frequency components).

Another interesting result in Figure 6 is that the spectra show several sharp peaks superimposed to a nearly constant trend versus $T$. These peaks correspond to periods where the uncontrolled spectra do not experience significant dynamic amplification effects, i.e. where the structure is not sufficiently "tuned" to any of the 36 motions and therefore needs lesser protection. If an infinite number of motions had been used instead of the 36 considered here, these spectra would have had their peaks smoothed and would have approached the nearly constant trend underneath, making TMD performance look more favorable. This suggests the following criterion to condense along the $T$ axis each percentile response ratio spectrum, in order to provide a synthetic (scalar) performance index: instead of simply averaging the response ratio along $T$, which would attribute an excessive weight to the said peaks, the average is computed of the inverse of the spectral ordinates, and the inverse of such average is assumed as the "performance index" (PI). This criterion, in fact weighing more the lower than the higher spectral ordinates, seems more adequate to catch the spectral-peak-smoothing effect, and will be used from here on.

By applying such criterion, the displacement, velocity and acceleration PIs are reported in Table 2 for 2 different percentiles, namely $50 \%$ and $95 \%$. Because of the bad matching observed for $T<1.5 \mathrm{~s}$, the average is restricted to the range $T>1.5 \mathrm{~s}$ for the analytical motions. For the real records, the PIs are computed separately for $T$ $<1.5 \mathrm{~s}$ and for $T>1.5 \mathrm{~s}$.

Table 2 suggests the following conclusions, valid for SDOF structures:

(a) TMDs effectiveness improves with increasing the percentile of response. Such robustness towards the severity of seismic effects is a fundamental property of TMDs, reflecting their ability to intervene when the structure is more closely "tuned" to the earthquake. This trend is more evident for the analytical pulses than for the real records, as a result of the analytical pulses possessing a narrower frequency band. The $95 \%$ is of particular interest because assumed by many building codes worldwide as a reference value for the definition of design loads;

(b) Focusing on the $95 \%$ percentile, for $T>1.5 \mathrm{~s}$ the $\mathrm{H}_{p}{ }^{*}$ method is slightly superior to the $\mathrm{H}_{\infty}$ method in terms of $R_{d}$ but inferior in terms of $R_{A}$ and particularly of $R_{v}$. For $T<1.5 \mathrm{~s}$ (where the analytical model becomes inadequate), the $\mathrm{H}_{p}{ }^{*}$ method (relying on that model) becomes inferior even in terms of $R_{d}$. As to the (practically inapplicable) $\mathrm{H}_{p}$ design, it does not show any significant advantage over the $\mathrm{H}_{p}{ }^{*}$ method, and will no longer be insisted upon in the sequel. In short, as the transfer functions are replaced by the response spectra and real records are 
considered, the trade-off between $\mathrm{H}_{p}{ }^{*}$ and $\mathrm{H}_{\infty}$ shifts in favor of the latter, and the $\mathrm{H}_{p}{ }^{*}$ method keeps preferable only for peak displacement reduction of flexible structures;

(c) Focusing on the $95 \%$ percentile, analytical ground motions portrait a TMD performance close to that obtained using real records. A slight performance overestimation is provided by the analytical model only in certain cases, with a maximum discrepancy of about $10 \%$ for $R_{v}$ when the $\mathrm{H}_{\infty}$ method is applied. If the $50 \%$ percentile is considered, conversely, the analytical model systematically underestimates performance in the order of a $10 \%$. It can be concluded that the analytical pulse model is an acceptable tool for the assessment of TMD performance on SDOF structures subjected to pulse-like records, particularly if large percentiles are of interest;

(d) For real records, TMD effectiveness results on average about $10-15 \%$ larger for $T<1.5 \mathrm{~s}$ than for $T>1.5 \mathrm{~s}$;

(e) Focusing on the $95 \%$ percentile, and averaging between the two design methods as well as between real and analytical inputs, the following PIs are obtained: for $\mu=10 \%, R_{d}=0.79, R_{v}=0.62$, and $R_{A}=0.76$; for $\mu=50 \%, R_{d}=0.65$, $R_{v}=0.43$, and $R_{A}=0.60$. The possibility of extending such conclusions to MDOF structures will be considered in the next section.

\begin{tabular}{|c|c|c|c|c|c|c|c|c|}
\hline \multirow[b]{2}{*}{$\mu$} & \multirow{2}{*}{$\begin{array}{l}\text { Design } \\
\text { method }\end{array}$} & \multirow{2}{*}{$T$ range } & \multicolumn{2}{|c|}{$R_{d}$} & \multicolumn{2}{|c|}{$K_{v}$} & \multicolumn{2}{|c|}{$R_{A}$} \\
\hline & & & $50 \%$ & $95 \%$ & $50 \%$ & $95 \%$ & $50 \%$ & $95 \%$ \\
\hline \multirow{9}{*}{$10 \%$} & \multirow{3}{*}{$\mathrm{H}_{\infty}$} & Rec. $<1.5 \mathrm{~s}$ & 0.80 & 0.73 & 0.51 & 0.44 & 0.75 & 0.68 \\
\hline & & Rec. $>1.5 \mathrm{~s}$ & 0.88 & 0.83 & 0.69 & 0.49 & 0.88 & 0.79 \\
\hline & & Anal. $>1.5 \mathrm{~s}$ & 0.97 & 0.81 & 0.75 & 0.45 & 0.95 & 0.77 \\
\hline & \multirow{4}{*}{$\mathrm{H}_{p}$} & Rec. $<1.5 \mathrm{~s}$ & 0.85 & 0.78 & 0.75 & 0.63 & 0.81 & 0.75 \\
\hline & & Rec. $>1.5 \mathrm{~s}$ & 0.88 & 0.82 & 0.85 & 0.75 & 0.91 & 0.84 \\
\hline & & Anal. $>1.5 \mathrm{~s}$ & 0.96 & 0.77 & 0.93 & 0.76 & 0.97 & 0.79 \\
\hline & & Rec. $<1.5 \mathrm{~s}$ & 0.85 & 0.80 & 0.71 & 0.65 & 0.82 & 0.77 \\
\hline & \multirow{2}{*}{$\mathrm{H}_{p}{ }^{*}$} & Rec. $>1.5 \mathrm{~s}$ & 0.88 & 0.80 & 0.83 & 0.67 & 0.91 & 0.80 \\
\hline & & Anal. $>1.5 \mathrm{~s}$ & 0.96 & 0.77 & 0.90 & 0.65 & 0.96 & 0.77 \\
\hline \multirow{9}{*}{$50 \%$} & \multirow{3}{*}{$\mathrm{H}_{\infty}$} & Rec. $<1.5 \mathrm{~s}$ & 0.79 & 0.62 & 0.41 & 0.32 & 0.61 & 0.52 \\
\hline & & Rec. $>1.5 \mathrm{~s}$ & 0.81 & 0.68 & 0.58 & 0.37 & 0.75 & 0.59 \\
\hline & & Anal. $>1.5 \mathrm{~s}$ & 0.89 & 0.66 & 0.69 & 0.32 & 0.81 & 0.57 \\
\hline & \multirow{4}{*}{$\mathrm{H}_{p}$} & Rec. $<1.5 \mathrm{~s}$ & 0.76 & 0.62 & 0.46 & 0.37 & 0.66 & 0.57 \\
\hline & & Rec. $>1.5 \mathrm{~s}$ & 0.81 & 0.69 & 0.64 & 0.46 & 0.82 & 0.71 \\
\hline & & Anal. $>1.5 \mathrm{~s}$ & 0.91 & 0.66 & 0.71 & 0.41 & 0.89 & 0.69 \\
\hline & & Rec. $<1.5 \mathrm{~s}$ & 0.76 & 0.62 & 0.44 & 0.37 & 0.66 & 0.59 \\
\hline & \multirow{2}{*}{$\mathrm{H}_{p}{ }^{*}$} & Rec. $>1.5 \mathrm{~s}$ & 0.81 & 0.67 & 0.64 & 0.43 & 0.83 & 0.69 \\
\hline & & Anal. $>1.5 \mathrm{~s}$ & 0.91 & 0.65 & 0.71 & 0.38 & 0.88 & 0.66 \\
\hline
\end{tabular}

Table 2: PIs for the SDOF structure under the recorded (Rec.) or analytical (Anal.) pulse ground motions in Set-36 


\section{Performance of TMDs on MDOF structures under pulse-like ground motions}

TMDs assessment, so far limited to SDOF systems, is here extended to a planar 10storey shear-type building structure. The structure has a constant mass on every floor, a linearly decreasing inter-storey stiffness along the height (with the base stiffness being 4 times the top stiffness), and 2\% damping ratio in every mode. The second and third eigenfrequencies are respectively 2.57 and 4.14 times the first one; and the first, second and third modal masses are respectively $77.5 \%, 12.2 \%$ and $4.4 \%$ the total mass of the structure. As for the SDOF case, the first period $T$ of the MDOF building is varied from 0 to 10 s providing response spectra. The TMD, placed on the top storey, is tuned to the first mode of the structure (the target mode), according to alternatively the $\mathrm{H}_{\infty}$ or the $\mathrm{H}_{p}{ }^{*}$ methods.

These methods, conceived for SDOF structures, can be adapted to MDOF structures through, for instance, the mass ratio equivalence proposed by Warburton [8]. The latter states that, as far as the target mode is sufficiently far from other modes, the TMD can be designed on an MDOF structure as for an SDOF structure, i.e. TMD optimum parameters $r$ (referred now to the frequency of the target mode) and $\zeta_{t}$ are unchanged, provided that the definition of the mass ratio is properly adjusted for the MDOF structure. Namely, the (effective) mass ratio $\mu$ will now denote the ratio of the TMD mass to the effective modal mass of the target mode, $m_{\text {leff, }}$ on its turn defined as the modal mass of the target mode, $m_{l}$, divided by the amplitude, $\phi_{1 t o p}$, of the mass-normalized modeshape of the target mode at the top floor (where the TMD is placed). As a result, $\mu$ is generally larger than the total mass ratio $\mu_{t o t}$, defined on its turn as the ratio of the TMD mass to the total structural mass.

In this section, the TMD is assigned the same (effective) mass ratios (and thus the same values of $r$ and $\zeta_{t}$ ) used for the SDOF case, i.e. $\mu=10 \%$ and $\mu=50 \%$, respectively corresponding to $\mu_{t o t}=4.06 \%$ and $\mu_{t o t}=20.3 \%$. In other words, according to Warburton's equivalence, a tuned mass equaling $4 \%$ of the total mass of the MDOF building is expected to reduce the structural response in the first mode as a $10 \%$ mass ratio TMD would do for an SDOF system, once the same optimal parameters are used. The MDOF/TMD combined system is then tested under the ground motions in Set-36, and percentile spectra are computed as for the SDOF case, expressed now in terms of 6 different structural responses: maximum peak relative displacement $d$; square root of the viscous energy dissipated during the earthquake by the structure, intended as a weighted measure of RMS velocities and then denoted as $v$; maximum peak absolute acceleration $A$; maximum peak interstorey drift ratio $d_{r}$; peak base shear force $S$; peak base bending moment $M$. The 95\% percentile response ratio spectra obtained applying the $\mathrm{H}_{p}{ }^{*}$ design, reported only in terms of $d, v$, and $A$ for brevity's sake, are given in Figure 6, superimposed to those corresponding to the SDOF case. The PIs for both the $\mathrm{H}_{\infty}$ and the $\mathrm{H}_{p}{ }^{*}$ methods are given in Table 3, which is the MDOF equivalent of Table 2. 


\begin{tabular}{|c|c|c|c|c|c|c|c|c|}
\hline \multirow{2}{*}{$\mu$} & \multirow{2}{*}{$\begin{array}{l}\text { Design } \\
\text { method }\end{array}$} & \multirow{2}{*}{$T$ range } & \multicolumn{2}{|c|}{$R_{d}$} & \multicolumn{2}{|c|}{$R_{v}$} & \multicolumn{2}{|c|}{$R_{A}$} \\
\hline & & & $50 \%$ & $95 \%$ & $50 \%$ & $95 \%$ & $50 \%$ & $95 \%$ \\
\hline \multirow{6}{*}{$10 \%$} & \multirow{3}{*}{$\mathrm{H}_{\infty}$} & Rec. $<1.5 \mathrm{~s}$ & 0.79 & 0.74 & 0.56 & 0.47 & 0.77 & 0.70 \\
\hline & & Rec & 0.86 & 0.81 & 0.78 & 0.59 & 0.94 & 0.94 \\
\hline & & Anal. $>1.5 \mathrm{~s}$ & 0.93 & 0.81 & 0.73 & 0.47 & 0.89 & 0.94 \\
\hline & \multirow{3}{*}{$\mathrm{H}_{p}{ }^{*}$} & Rec. $<1.5 \mathrm{~s}$ & 0.85 & 0.82 & 0.76 & 0.68 & 0.84 & 0.82 \\
\hline & & Rec. $>1.5 \mathrm{~s}$ & 0.88 & 0.80 & 0.90 & 0.76 & 0.97 & 0.97 \\
\hline & & Anal. $>1.5 \mathrm{~s}$ & 0.95 & 0.77 & 0.92 & 0.69 & 0.90 & 0.97 \\
\hline \multirow{6}{*}{$50 \%$} & \multirow{3}{*}{$\mathrm{H}_{\infty}$} & Rec. & 0.75 & 0.61 & 0.42 & 0.33 & 0.61 & 0.52 \\
\hline & & Rec. $>1.5 \mathrm{~s}$ & 0.71 & 0.64 & 0.61 & 0.43 & 0.82 & 0.80 \\
\hline & & Anal. $>1.5 \mathrm{~s}$ & 0.80 & 0.64 & 0.56 & 0.34 & 0.71 & 0.80 \\
\hline & \multirow{3}{*}{$\mathrm{H}_{p}{ }^{*}$} & Rec. $<1.5 \mathrm{~s}$ & 0.74 & 0.63 & 0.49 & 0.41 & 0.68 & 0.61 \\
\hline & & Rec. $>1.5 \mathrm{~s}$ & 0.76 & 0.68 & 0.70 & 0.51 & 0.89 & 0.87 \\
\hline & & Anal. $>1.5 \mathrm{~s}$ & 0.85 & 0.66 & 0.64 & 0.42 & 0.79 & 0.86 \\
\hline
\end{tabular}

Table 3: PIs for the MDOF structure under the recorded (Rec.) or analytical (Anal.) pulse ground motions in Set-36

Figure 6 provides MDOF spectra which are very similar to the SDOF ones, except for the accelerations, whose curves rapidly converges to unity beyond a certain period, as soon as uncontrolled higher modal responses become prevalent.

Comparing Table 3 with Table 2, it can be observed that:

(a) $R_{d}$ is unchanged for the MDOF case with respect to the SDOF case;

(b) $R_{v}$ increases for the MDOF case, although at different extents: for $T<1.5 \mathrm{~s}, R_{v}$ is little affected (the first mode is still prevalent); for $T>1.5 \mathrm{~s}, R_{v}$ increases about 15$20 \%$ under real records, but only about $6 \%$ under analytical pulses (which excite less the higher modes due to their lacking of high frequency components), so that the discrepancy between real records and analytical pulses augments, reaching a maximum of $25 \%$ (instead of merely $10 \%$ ), with the pulse model systematically overestimating TMD performance;

(c) $R_{A}$ increases even more than $R_{v}$, typically about $15-25 \%$; but since this happens for both real records and analytical pulses, the discrepancy between them remains small.

In conclusion, as far as the peak displacement or peak accelerations are considered, the analytical approximation works for the MDOF structure as well as for the SDOF structure; for the RMS velocity, it overestimates TMD benefits. Also, the TMD is still effective to reduce $d$ and $v$, but scarcely effective against $A$ for medium-to-long periods. The $\mathrm{H}_{\infty}$ and the $\mathrm{H}_{p}{ }^{*}$ methods are comparable in reducing $d$, but $\mathrm{H}_{\infty}$ is superior in reducing $A$ and even more $v$.

Not shown for brevity, results for the base shear force $S$ and the base bending moment $M$ are very similar to those reported above for $d$, while results for the drift ratio $d_{r}$ are somewhat intermediate between those obtained for $d$ and for $A$. 


\section{Validation on a larger set of 166 real records with impulsive features}

To validate previous results on a larger set of real records, the $\mathrm{H}_{\infty}$ and the $\mathrm{H}_{p}{ }^{*}$ strategies are tested under 166 near-field records (including the 36 used above). Comparison with the analytical model is here abandoned. The 166 records, herein termed Set-166, are assembled through merging Set-36 with the following two sets, identified in the literature as representative of pulse-like NF ground motions (and characterized by moment magnitude $M_{w}>5.6$ ):

- Set-140: 70 ground-motion pairs (140 components) assembled in [9].

- Set-104: 52 ground-motion pairs (104 components) used in [2].

The resulting 166 records, downloaded from the PEER NGA strong-motion database, are here used with no further correction processing (neither rotation nor amplitude scaling), so as to provide an ensemble of records whose amplitude distribution is, grossly speaking, statistically representative of the seismic hazard for a building located in a generic NF zone (for the chosen magnitude interval).

Results are presented in Figure 7 (for the $\mathrm{H}_{\infty}$ design only) and in Table 4 (comparing $\mathrm{H}_{\infty}$ and $\mathrm{H}_{p}{ }^{*}$ options), for both the SDOF and the MDOF cases.

Figure 7, compared with Figure 6, testifies the beneficial smoothing effect obtained enlarging the set of records.

Comparing Table 4 with Tables 2 and 3, a substantial coherence can be appreciated between Set-36 and Set-166. Dividing, cell by cell, Table 4 by the corresponding values of Tables 2 and 3, and then taking the average, a value of 1.00 is obtained. Not reported for brevity, similar results are obtained for $d_{r}, S$ and $M$.
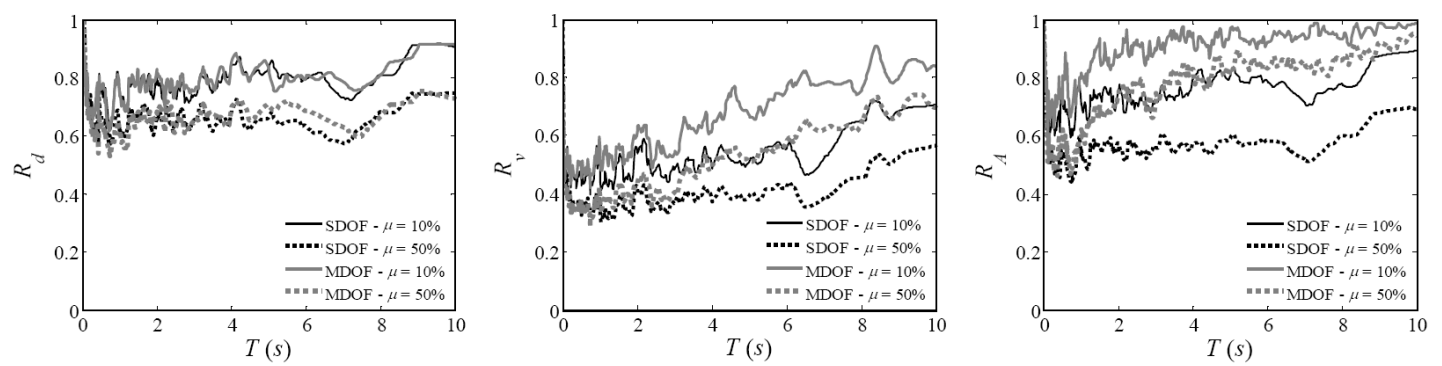

Figure 7: 95\% percentile response ratio spectra under the real records in

Set-166, for SDOF and MDOF structures controlled through an $\mathrm{H}_{\infty}$-designed TMD $(\mu=10 \%$ or $\mu=50 \%)$

It can be concluded that the results obtained in previous sections for the set of 36 ground motions are generally valid for a larger set of typical impulsive ground motions. With the limitations pointed out in previous sections, the set of 36 analytical ground motions is therefore adequate to statistically assess TMDs effectiveness under impulsive records. That is, to the purpose of TMD evaluation, the analytical model in Eqs. (1) to (2) is an useful assessment tool and the $36 \mathrm{~N}, \zeta_{p}$ pairs are well distributed so as to be statistically representative. 


\begin{tabular}{|c|c|c|c|c|c|c|c|c|}
\hline \multirow[b]{2}{*}{$\mu$} & \multirow{2}{*}{$\begin{array}{l}\text { Design } \\
\text { method }\end{array}$} & \multirow{2}{*}{$T$ range } & \multicolumn{2}{|c|}{$R_{d}$} & \multicolumn{2}{|c|}{$R_{v}$} & \multicolumn{2}{|c|}{$R_{A}$} \\
\hline & & & $50 \%$ & $95 \%$ & $50 \%$ & $95 \%$ & $50 \%$ & $95 \%$ \\
\hline \multirow{8}{*}{$10 \%$} & \multirow{5}{*}{$\mathrm{H}_{\infty}$} & $\mathrm{SDOF}<1.5 \mathrm{~s}$ & 0.78 & 0.73 & 0.51 & 0.46 & 0.73 & 0.68 \\
\hline & & $\mathrm{SDOF}>1.5 \mathrm{~s}$ & 0.83 & 0.81 & 0.71 & 0.55 & 0.82 & 0.78 \\
\hline & & $\mathrm{MDOF}<1.5 \mathrm{~s}$ & 0.78 & 0.73 & 0.55 & 0.50 & 0.79 & 0.77 \\
\hline & & $\mathrm{MDOF}>1.5 \mathrm{~s}$ & 0.84 & 0.81 & 0.79 & 0.70 & 0.95 & 0.94 \\
\hline & & $\mathrm{SDOF}<1.5 \mathrm{~s}$ & 0.85 & 0.78 & 0.71 & 0.67 & 0.82 & 0.75 \\
\hline & \multirow{3}{*}{$\mathrm{H}_{p}{ }^{*}$} & $\mathrm{SDOF}>1.5 \mathrm{~s}$ & 0.84 & 0.79 & 0.82 & 0.71 & 0.87 & 0.82 \\
\hline & & MDOF $<1.5 \mathrm{~s}$ & 0.85 & 0.77 & 0.75 & 0.70 & 0.87 & 0.86 \\
\hline & & $\mathrm{MDOF}>1.5 \mathrm{~s}$ & 0.87 & 0.82 & 0.90 & 0.85 & 0.98 & 0.97 \\
\hline \multirow{8}{*}{$50 \%$} & \multirow{4}{*}{$\mathrm{H}_{\infty}$} & $\mathrm{SDOF}<1.5 \mathrm{~s}$ & 0.72 & 0.65 & 0.40 & 0.35 & 0.57 & 0.52 \\
\hline & & $\mathrm{SDOF}>1.5 \mathrm{~s}$ & 0.72 & 0.66 & 0.61 & 0.42 & 0.67 & 0.58 \\
\hline & & $\mathrm{MDOF}<1.5 \mathrm{~s}$ & 0.70 & 0.62 & 0.40 & 0.36 & 0.60 & 0.57 \\
\hline & & $\mathrm{MDOF}>1.5 \mathrm{~s}$ & 0.69 & 0.68 & 0.62 & 0.54 & 0.85 & 0.82 \\
\hline & \multirow{4}{*}{$\mathrm{H}_{p}{ }^{*}$} & $\mathrm{SDOF}<1.5 \mathrm{~s}$ & 0.71 & 0.63 & 0.44 & 0.39 & 0.65 & 0.60 \\
\hline & & $\mathrm{SDOF}>1.5 \mathrm{~s}$ & 0.74 & 0.68 & 0.66 & 0.49 & 0.76 & 0.69 \\
\hline & & $\mathrm{MDOF}<1.5 \mathrm{~s}$ & 0.70 & 0.63 & 0.48 & 0.43 & 0.69 & 0.68 \\
\hline & & MDOF $>1.5 \mathrm{~s}$ & 0.74 & 0.71 & 0.70 & 0.62 & 0.90 & 0.88 \\
\hline
\end{tabular}

Table 4: PIs for SDOF and MDOF structures under the real records in Set-166

\section{Case-Study: a Roof-Garden TMD against Pulse-Like Ground Motions}

These conclusive pages suggest one possible solution for achieving the large mass ratios necessary for a TMD to effectively control impulsive earthquakes.

The example is the Roof-Garden TMD (RGTMD), proposed by the author to reduce the seismic response of a multi-storey building structure recently constructed in Siena, one of the most beautiful medieval towns in Central Italy. The building is the D Unit of the Portasiena Linear Building complex, a polyfunctional commercial centre on which roof-gardens of the "intensive" kind were prescribed for architectural reasons. A rendering view of the complex and an axonometric projection of the 3D FEM model for the D Unit are given in Figure 8.

Involved in the structural design of the complex, the author explored the idea of turning the additional mass of the roof-garden into an innovative TMD, capable of both environmental and seismic protection. This is achieved by adding a new floor atop the building, connected to the original top storey through a proper system of bearings and dashpots, and filled with planted soil.

A detailed description of the structure and of three possible schemes of RGTMD is given in [10]. The structure is schematized as a planar 6DOF model with 5\% damping in every mode. The three lower modes have natural frequencies $2.35 \mathrm{~Hz}$, $9.29 \mathrm{~Hz}$ and $18.9 \mathrm{~Hz}$ and modal mass ratios $57.9 \%, 25.4 \%$ and $9.3 \%$. The mass of the RGTMD $\left(1270 \mathrm{~kg} / \mathrm{m}^{2}\right)$ is $17.1 \%$ the mass of the building $\left(\mu_{t o t}=17.1 \%\right)$, 
corresponding to an "effective mass ratio" $\mu=76.3 \%$. The $\mathrm{H}_{\infty}$ design is applied by numerically minimizing the peak modulus of the transfer function from the ground acceleration to the maximum interstorey drift ratio, resulting in the optimal parameters $r=0.421$ and $\zeta_{t}=0.523$.

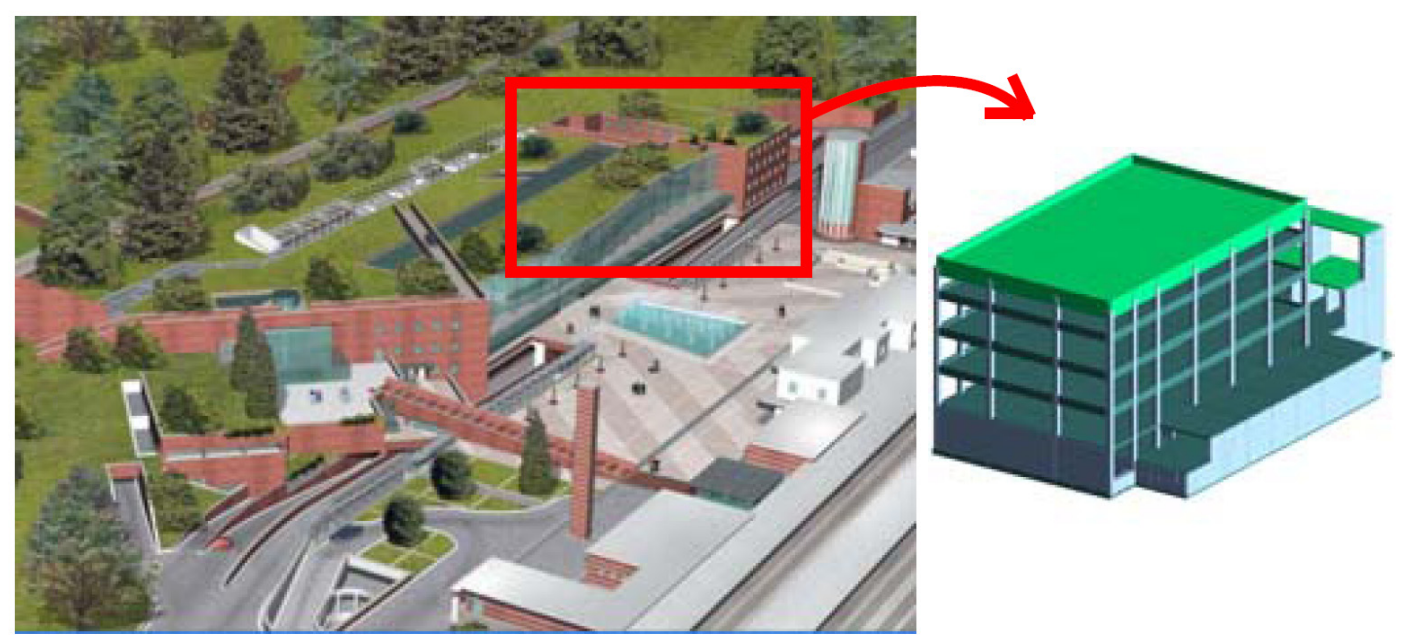

Figure 8: Simulation of an RGTMD in Portasiena Linear Building: (left) architectural rendering of the complex; (right) FEM model of the D Unit

With and without the TMD atop, the structure is simulated under the records in Set166. The satisfactory performance of the RGTMD is confirmed by Table 5, which compares the $50 \%$ and the $95 \%$ percentiles of interesting peak responses. Looking in particular at the $95 \%$ percentile, the maximum displacement $d$ and the maximum interstorey drift ratio $d_{r}$ drastically drop to, respectively, $57.6 \%$ and $54.1 \%$ if the RGTMD is installed; the maximum acceleration $A$ only reduces to $94.4 \%$ due to the effect of uncontrolled higher modes; the reduction of the base shear force $S$ is someway intermediate $(73.1 \%)$. Finally, as a remarkable byproduct of deploying a large mass ratio, the $95 \%$ percentile of the TMD peak displacement relative to the structure, $d_{T M D}$, is limited to $11.1 \mathrm{~cm}$, which is compatible with a variety of constructive options for the TMD bearing system, including the simple and costeffective rubber bearing arrangement suggested in [10].

\begin{tabular}{|c|c|c|c|c|c|c|}
\hline Percentile & Case & $\begin{array}{c}d_{r} \\
(\%)\end{array}$ & $\begin{array}{c}d \\
(\mathrm{~cm})\end{array}$ & $\begin{array}{c}A \\
\left(\mathrm{~m} / \mathrm{s}^{2}\right)\end{array}$ & $\begin{array}{c}S \\
(\mathrm{MN})\end{array}$ & $\begin{array}{c}d_{T M D} \\
(\mathrm{~cm})\end{array}$ \\
\hline \multirow{2}{*}{$50 \%$} & Unc. & 0.306 & 4.49 & 10.7 & 50.7 & - \\
& Contr. & 0.173 & 2.60 & 8.63 & 35.3 & 4.82 \\
\hline \multirow{2}{*}{$95 \%$} & Unc. & 0.764 & 11.0 & 25.6 & 116 & - \\
& Contr. & 0.413 & 6.33 & 24.2 & 84.7 & 11.1 \\
\hline
\end{tabular}

Table 5: Peak seismic responses of the D Unit under the real records in Set-166 


\section{Conclusions}

The paper investigates the effectiveness of TMDs against impulsive seismic excitations. In order to explore the possible advantages of a pulse-oriented TMD design, the analytical pulse model by He and Agrawal is adopted, and an extension of the classical $\mathrm{H}_{\infty}$ design is proposed, termed $\mathrm{H}_{p}$, which minimizes the peak structural response to a given analytical pulse. This new $\mathrm{H}_{p}$ design, by virtue of a reduced damping ratio, improves control in peak terms, but worsens it in RMS terms. Also, since it presumes the exact knowledge of future pulses, it is impractical in reality. The $\mathrm{H}_{p}{ }^{*}$ variant is thus introduced which, merely relying on an average estimate of ground motion features, shows a pulse performance only slightly inferior to that of $\mathrm{H}_{p}$, resulting on the other hand a directly useable design tool.

The $\mathrm{H}_{\infty}$ and the $\mathrm{H}_{p}{ }^{*}$ methods are then tested on SDOF and MDOF structures under real records and analytical pulses, in order to: (a) assess the accuracy of the pulse model as a design tool; and (b) compare the two design approaches. Percentile response spectra (and their condensation into performance indices) are introduced as the natural tool to evaluate a control strategy which proves "more effective when more needed", and a large set of real records is used to achieve statistical consistency. In order for the TMD to attain a satisfactory performance, mass ratios are tried which are much larger than usual but still practicable, for instance recurring to the concept of the Roof-Garden TMD, recently proposed by the author.

As a result of this work, conclusions can be summarized as follows:

1. TMDs can be effective even against impulsive loads if large mass ratios are used; the effectiveness, larger in RMS than in peak terms, increases with the percentile of structural response, indicating TMD robustness towards the severity of seismic effects;

2. As far as peak displacement is concerned, the effectiveness of a TMD on an SDOF structure is similar to that of a TMD of equal "effective" mass ratio on an MDOF structure; but as RMS velocity or peak acceleration are considered, the effectiveness in the MDOF case decreases with an increase in the structural period as a result of uncontrolled higher modes, while for the SDOF case it keeps nearly constant;

3. If real records are considered, the $\mathrm{H}_{p}{ }^{*}$ design surpasses the $\mathrm{H}_{\infty}$ design in reducing the peak displacement of SDOF systems; in all other cases the $\mathrm{H}_{\infty}$ design seems generally preferable; the advantages of taking into account the impulsiveness of the ground motion directly at the design stage are therefore circumscribed to very peculiar cases, and the $\mathrm{H}_{\infty}$ method can be generally recommended as a design tool;

4. As long as the structural period is sufficiently large (i.e. close to or longer than the pulse period), the analytical pulse model proves an accurate and efficient tool for the assessment of TMDs under impulsive ground motions.

Extending the scope of the present study to the case of nonlinear structural systems will be the object of a future work. 


\section{References}

[1] A.M. Kaynia, D. Veneziano, J.M. Biggs, "Seismic effectiveness of tuned mass dampers", Journal of Structural Engineering, 107(8), 1465-1484, 1981.

[2] J.D. Bray, A. Rodriguez-Marek, "Characterization of forward-directivity ground motions in the near-fault region", Soil Dynamics and Earthquake Engineering, 24, 815-828, 2004.

[3] N. Makris, S.P. Chang, "Response of damped oscillators to cycloidal pulses", Journal of Engineering Mechanics, 126(2), 123-131, 2000.

[4] B. Alavi, H. Krawinkler, "Effects of near-fault ground motions on frame structures", The John A. Blume Earthquake Engineering Center Research Rep. No. 138, Stanford, California, 2001.

[5] M. Dicleli, S. Buddaram, "Equivalent linear analysis of seismic-isolated bridges subjected to near-fault ground motions with forward rupture directivity effect", Engineering Structures, 29, 21-32, 2007.

[6] W.L. He, A.K. Agrawal, "Analytical model of ground motion pulses for the design and assessment of seismic protective systems", Journal of Structural Engineering, 134(7), 1177-1188, 2008.

[7] N. Hoang, Y. Fujino, P. Warnitchai, "Optimal tuned mass damper for seismic applications and practical design formulas", Engineering Structures, 30, 707$715,2008$.

[8] G.B. Warburton, "Optimum absorber parameters for various combinations of response and excitation parameters." Earthquake Engineering and Structural Dynamics, 10, 381-401, 1982.

[9] P. Tothong, C.A. Cornell, "Probabilistic seismic demand analysis using advanced ground motion intensity measures, attenuation relationships, and near-fault effects." PEER Report 2006/11, Berkeley, California, 2007.

[10] E. Matta, A. De Stefano, "Seismic performance of pendulum and translational roof-garden TMDs.” Mechanical Systems and Signal Processing, 23, 908-921, 2009. 
2012-12-01

\section{Tuned mass dampers for the mitigation of impulsive ground motions}

Matta, Emiliano

Civil-Comp Press

Emiliano Matta, Rosario Ceravolo, Alessandro De Stefano, Antonino Quattrone and Luca

Zanotti Fragonara. Tuned mass dampers for the mitigation of impulsive ground motions. 11th International Conference on Computational Structures Technology 2012 (CST 2012), 4-7 September 2012, Dubrovnik, Croatia.

https://dspace.lib.cranfield.ac.uk/handle/1826/12258

Downloaded from Cranfield Library Services E-Repository 